\title{
A method for the prediction of relative sunspot number for the remainder of a progressing cycle with application to cycle 23
}

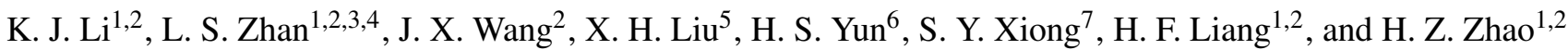 \\ 1 Yunnan Observatory, YN650011, China \\ 2 National Astronomical Observatories, CAS, China \\ 3 Academy of Graduates, CAS, Beijing, China \\ ${ }^{4}$ Department of Physics, Jingdezhen Comprehensive College, Jiangxi, China \\ 5 Department of Physics, Jiaozuo Educational College, Henan, China \\ 6 Astronomy Program, SEES, Seoul National University, Seoul, Korea \\ 7 Library, Yunnan Observatory, Yunnan, China
}

Received 28 May 2001 / Accepted 18 April 2002

\begin{abstract}
In this paper, we investigate the prospect of using previously occurring sunspot cycle signatures to determine future behavior in an ongoing cycle, with specific application to cycle 23, the current sunspot cycle. We find that the gross level of solar activity (i.e., the sum of the total number of sunspots over the course of a sunspot cycle) associated with cycle 23, based on a comparison of its first several years of activity against similar periods of preceding cycles, is such that cycle 23 best compares to cycle 2. Compared to cycles 2 and 22, respectively, cycle 23 appears 1.08 times larger and 0.75 times as large. Because cycle 2 was of shorter period, we infer that cycle 23 also might be of shorter length (period less than 11 years), ending sometime in late 2006 or early 2007.
\end{abstract}

Key words. Sun: activity - Sun: sunspots - Sun: general

\section{Introduction}

The sunspot activity is usually expressed in the Wolf sunspot index number (or sunspot numbers). Although the Wolf number $R$, involving spot counts by eye, is a subjective measure, moreover, its definition seems to be rather arbitrary, it serves in many solar activity studies, because it is the only record that covers more than two centuries with rather consistent data and correlates surprisingly well with more objective, quantitative indices, such as $10.7-\mathrm{cm}$ radio flux, total magnetic flux (Schrijver \& Zwaan 2000). Usually monthly averages or smoothed mean values of the Wolf number $R$ are used to take out the modulation by solar rotation and short-period fluctuations in solar activity.

The well-known 11-year period associated with sunspot cycles was first discovered by Schwabe (1843), then reported again by Wolf (1852) from his examination of the Zurich Observatory sunspot records. Waldmeier $(1935,1939)$ noted the asymmetry between the rise to maximum and fall to minimum as well as the importance of the amplitude in determining

Send offprint requests to: $\mathrm{K}$. J. Li, e-mail: kejunli@netease.com the shape and length of the cycle (Hathaway et al. 1994). Wilson (1988) and Wilson et al. (1998) provides a useful description of other relationships found by examining the historical records of previous sunspot cycle. These findings are useful for predicting of solar activity. However, unfortunately, sunspot cycle has no strictly period. Cycles are observed to vary both in size and length, and periods of inactivity like the Maunder Minimum are known to have occurred (Hathaway et al. 1994). Since the year 1755 , the beginning of solar cycle 1, the sunspot cycle varies markedly in amplitude by at least a factor of 4 . Also the cycle length (counted from one sunspot minimum to the next) varies appreciably, between approximately 8 and 15 yr (Schrijver \& Zwaan 2000). Therefore, prediction of the sunspot number cycle is of great difficulty but deserves to be done due to the extreme importance for many applications, including telecommunications and satellite orbitography (Lantos 2000; $\mathrm{Li}$ et al. 2001). Various methods for estimating the relative timing and strength of cycle 23 are examined and summarized by Hathaway et al. (1999, 2001), Li et al. (2001), and Kane (2001). In the present work, investigated is the possibility of using the known sunspot numbers to predict the forthcoming solar activity of a progressing cycle. 


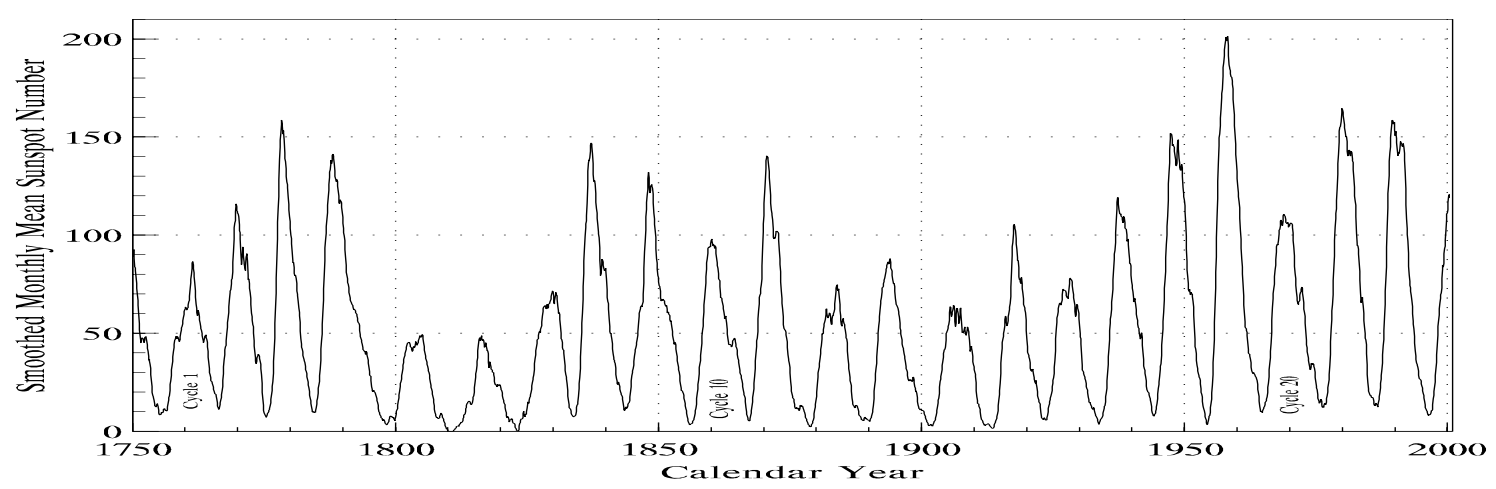

Fig. 1. Sunspot numbers of cycles 1 to 23 .

\section{Prospect of using the known cycles to predict solar activity of a progressing cycle}

The smoothed monthly mean values of the Wolf number $R$ of solar cycle 1 to the present are provided from the solar and upper atmosphere group at the National Geophysical Data Center (see the web site http: //www.ngdc.noaa.gov/stp/SOLAR/ getdata.html), which are shown in Fig. 1. The figure shows that cycles 1 to 22 are complete ones, but cycle 23 is progressing and uncompleted. For each of the last 6 complete cycles (cycles 17 to 22), which is called Part I here, we move the sunspot numbers $\left(R^{\mathrm{I}}(t)\right)$ of the cycle parallel along the time axis one cycle by one cycle toward each of the other 21 complete cycles (called Part II), whose sunspot numbers $\left(R^{\mathrm{II}}(t)\right)$ are multiplied by a coefficient $C$ in the meantime, until that the both cyclic series of the sunspot numbers have a least square difference. The two parameters, namely the time shift $T$ of moving Part I to Part II and the coefficient $C$ should make the both series finally have a least square difference with each other. The detailed procedure to get two expected parametric values is as follows. First, we estimate an approximate range of possible values of each of the two parameters. Then, given a series of values of the time shift $T$ ( $n$ values) and a series of values of the coefficient $C$ ( $m$ values) respectively at their ranges, we calculate the value of square difference of the both series within each set of parametric values. Finally, among the $n \times m$ values of the square difference we chose the set of parametric values, which gives a minimum value of the square difference $\left(\min \left\{\sum_{t}\left[C \times R^{\mathrm{II}}(t)-R^{\mathrm{I}}(t-T)\right]^{2}\right\}\right)$, as the best-fit result of Part I to Part II. Among the 21 complete cycles of Part II, the cycle, which has a minimum value of the square difference $\left(\min \left\{\sum_{t}\left[C \times R^{\mathrm{II}}(t)-R^{\mathrm{I}}(t-T)\right]^{2}\right\}\right)$ with the moved cycle (the certain Part I), is called the similar cycle of the moved cycle.

Next, we want to know whether the set of the two parametric values can be determined at the early stage of a Part I cycle. We delete the last one value of the Part I cycle and repeat the above procedure to get a best-fit result of Part I to Part II and obtain a set of parametric values. Then again, we delete the last two values of Part I and repeat the above procedure to get a best-fit result and obtain a set of parametric values, and so on until only twelve values of sunspot numbers left in Part I. Afterwards, we know the variation of the expected values of parameters $C$ and $T$ with the points of the used data of Part I when moving a Part I cycle toward the other Part II cycles.
Figures 2 and 3 show the variation of the determined parameters $C$ and $T$ with the used data points, respectively. There are 21 lines in each panel of the figures, corresponding to moving a Part I cycle toward the other 21 complete cycle, called as Part II cycles here.

Here, in fact, we choose a cycle as the similar cycle of a moved cycle according to the two criteria: the two cycles have a minimum value of the square difference $\left(\min \left\{\sum_{t}\left[C \times R^{\mathrm{II}}(t)-\right.\right.\right.$ $\left.\left.R^{\mathrm{I}}(t-T)\right]^{2}\right\}$ ) with each other when the given moved cycle progresses 3 to 4 years into cycle, and meantime, the determined parameters $C$ and $T$ have very small change, variation of parameter $C$ is less than $10 \%$, and parameter $T$ varies within 4 points (months).

Figures 4 and 5 respectively shows the determinations of parameters $C$ and $T$ at one-month time interval into the cycle of Part I when moving the cycle into its similar cycle. Concerning the time shift $T$, we have to point out that: at first we put the series of the sunspot numbers of the moved cycle at the end of a certain cycle of Part II, at this time the time shift $T$ is counted as zero, then the given cycle is moved into the certain cycle of Part II, and the time shift begins to count. Figures 4 and 5 indicate that the parameters $C$ and $T$ can actually be quite accurately determined within the first 2 to 3 years following the start of the given cycle (parameter $T$ can accurately determined about 0.5 year earlier than parameter $C$ ). This suggests that fitting the behavior of the given solar cycle with the known solar cycles after it progresses 3 to 4 years into cycle could find its similar cycle, and then give a good prediction of the behavior of the given solar cycle over the remaining 6 to 11 years of the cycle through its similar cycle.

Figure 6 displays comparison of the given cycles respectively with their similar cycles whose sunspot numbers are respectively multiplied by their corresponding determined parametric values $C$. The figure shows that the similar cycles indeed have their cycle shape resemble their corresponding moved cycle's shape after multiplied by a constant, the parameter $c$.

Here, we give a new index, the "weight center" of sunspot numbers of a cycle, which is defined as the time position: the total sunspot number before the time position is equal to that after. It is found that the time shift $T$ can accurately give the distant $(D)$ between two weight centers of the two involved cycles when moving one solar cycle toward the other. For cycles 17 to 22, the error between $T$ and its corresponding $D$ is not larger 

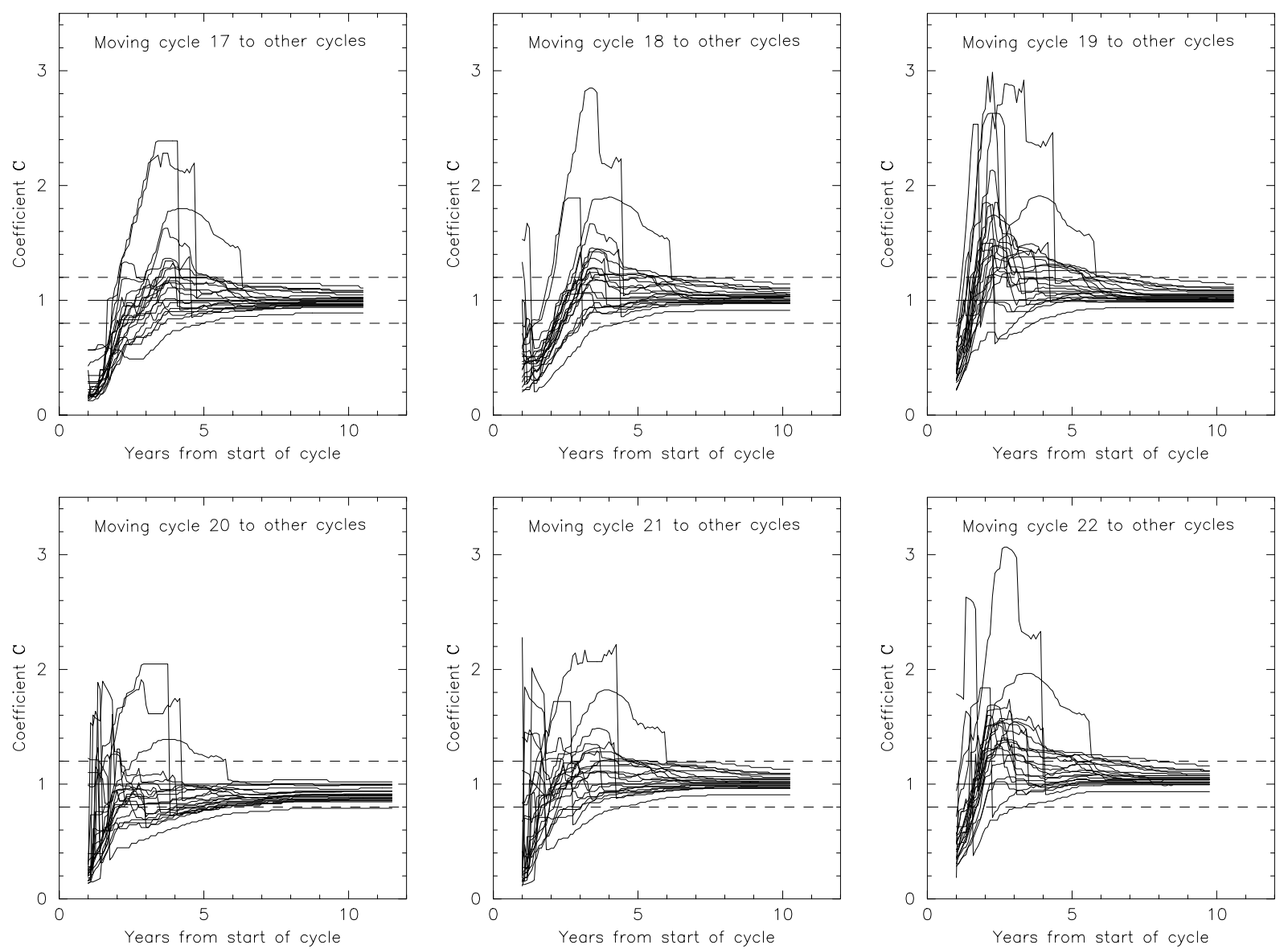

Fig. 2. Variation of the determined parameter $C$ with the used data points of a Part I cycle when moving the Part I cycle into its corresponding 21 Part II cycles.

than 2 months. For each of cycles 17 to 22 , we have calculated the ratio of total sunspot number of its similar cycle to that of its own cycle, resultingly, the ratios for cycles 17 to 22 are 1.10 , $1.26,1.15,1.87,1.10$, and 1.03 , respectively. Values of the parameter $C$ for cycles 17 to 22 are determined to be $1.15,1.30$, $1.17,1.77,1.09$, and 1.05 , respectively, so we know that the parameter $C$ can reflect the ratio of the two involved cycles with error less than $6 \%$, it means that we can accurately estimate the gross level (the total sunspot number) of solar activity of a cycle after 3 years following the beginning of the cycle. After understanding the implication of the two parameters, we can easily estimate an approximate range of their possible values: $8 \mathrm{yr} \leq T \leq 14 \mathrm{yr}$ and $0<C<8$.

\section{Prediction of the progressing solar cycle 23}

Conventionally, the beginning time of solar cycle 23 is regarded as May 1996, although from the perspective of curve fitting, it probably should be regarded as occurring slightly later in time. NOAA, for example, uses October 1996 as minimum for cycle 23 in their prediction of solar activity charts (Wilson et al. 1998; Harvey \& White 1999). Up to now, solar cycle 23 has progressed more than 4 years into cycle. With the use of the smoothed monthly mean values of the Wolf number $R$ of solar cycle 23 (from May 1996 to March 2001), we have determined the parameters $C$ and $T$ by using the method introduced in the last section. Figures 7 and 8 , respectively, show the variation of the determined parameters $C$ and $T$ with the used data points when moving cycle 23 toward the other 22 complete cycles, so, there are 22 lines in each of the two figures.

Among the 22 complete cycles, we found that cycle 2 is the similar cycle of cycle 23 . Figures 9 and 10 respectively show the determined parameters $C$ and $T$ at one-month time interval into cycle 23 when moving the cycle into its similar cycle. The gross level of solar activity of cycle 23 is estimated to be 1.08 times as much as cycle 2 and 0.75 times as much as its neighboring cycle, cycle 22. Comparison of cycle 23 and its similar cycle is shown in Fig. 11, which indicates that the similar cycle after multiplied by a constant can accurately describe the known part of solar activity of cycle 23 , so here we predict that solar activity of cycle 23 will develop into future along the trace of solar activity of the similar cycle, namely, the rest part of the similar cycle after March 2001 may be regarded as the developing tendency of solar cycle 23. MSFC Solar Physics Branch members Wilson, Hathaway, and Reichmann have studied the sunspot record for characteristic behavior that might help in predicting future sunspot activity and given a prediction of sunspot numbers of the current cycle. Their predicted numbers, which are available through web site http://www.science.nasa.gov/ssl/ pad/solar/predict . htm, are also plotted in Fig. 11. The figure shows that solar activity predicted by us fits well to the 

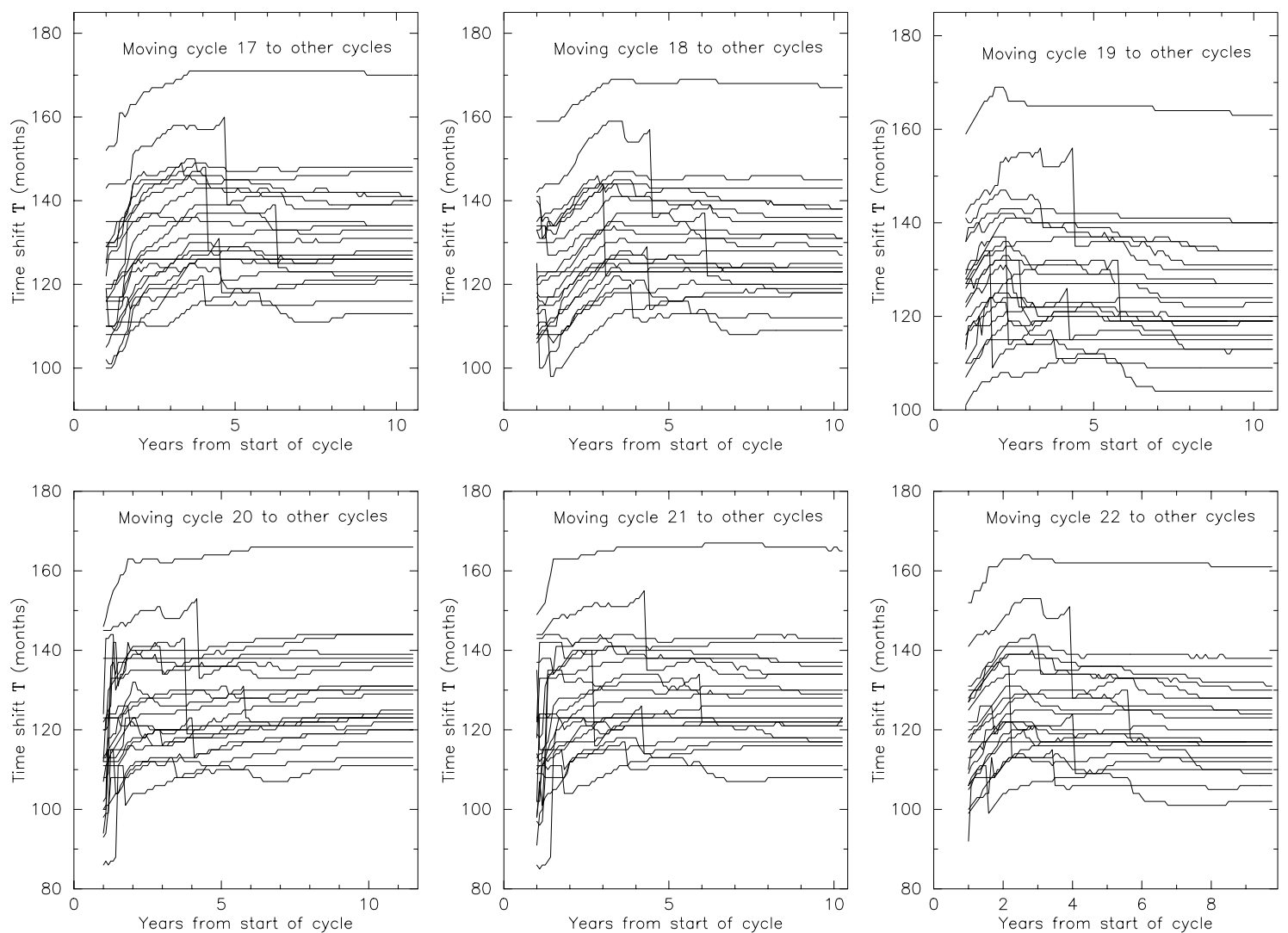

Fig. 3. Variation of the determined parameters $T$ with the used data points of a Part I cycle when moving the Part I cycle into its corresponding 21 Part II cycles.
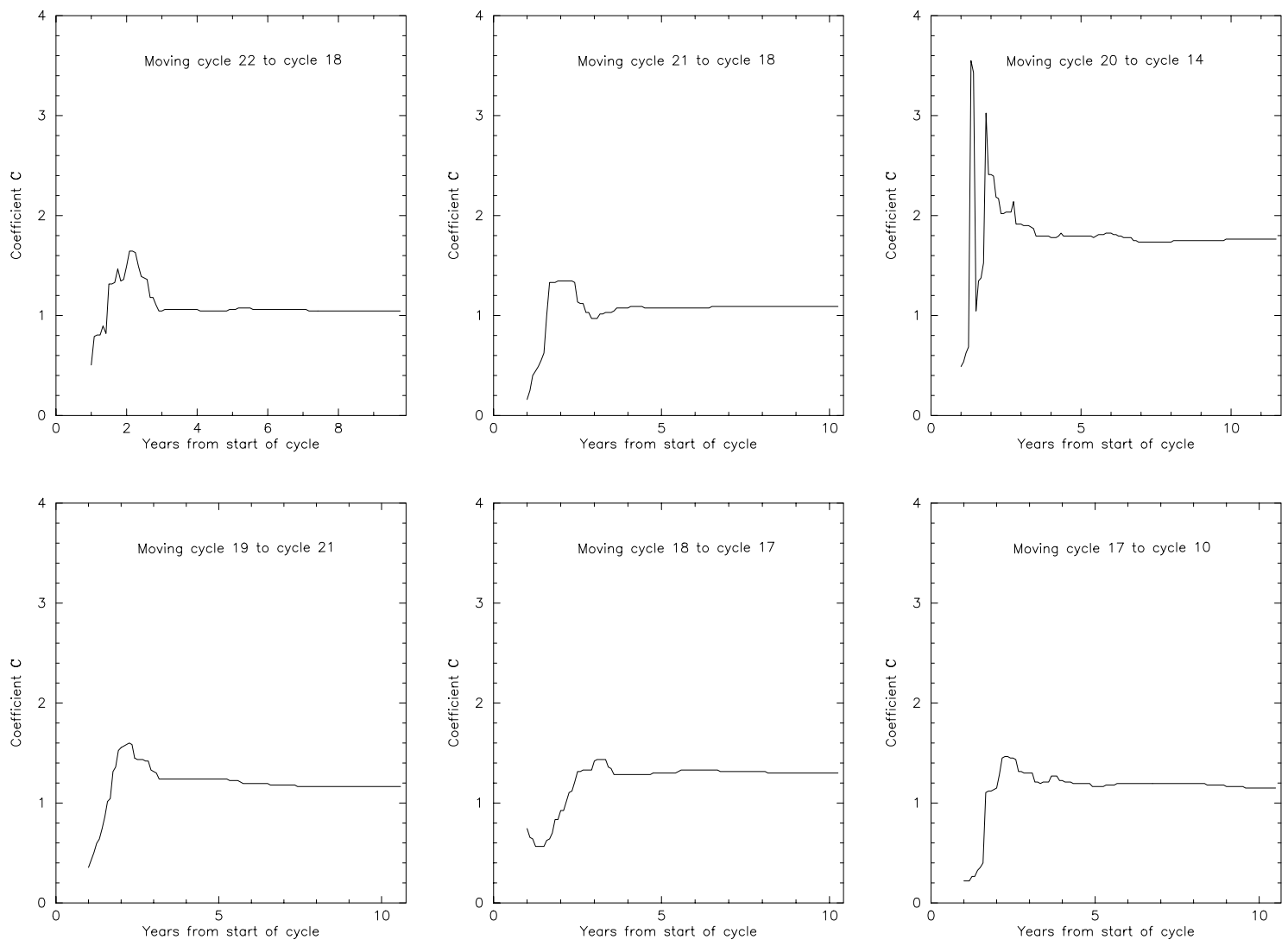

Fig. 4. Determinations of the parameter $C$ at one-month interval into each cycle of cycles 17 to 22 when moving them respectively into their similar cycles. 

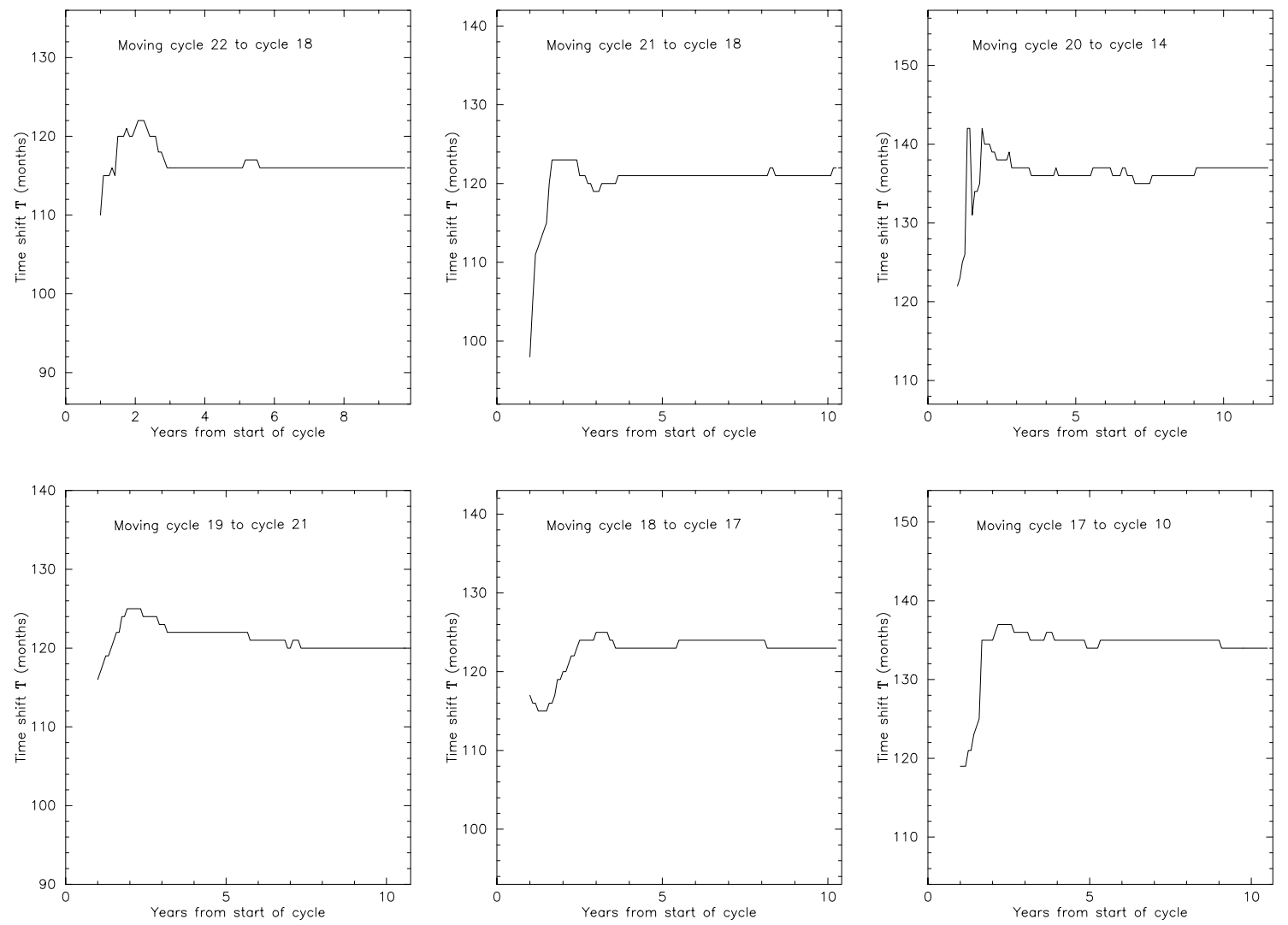

Fig. 5. Determinations of the parameter $T$ at one-month interval into each cycle of cycles 17 to 22 when moving them respectively into their similar cycles.
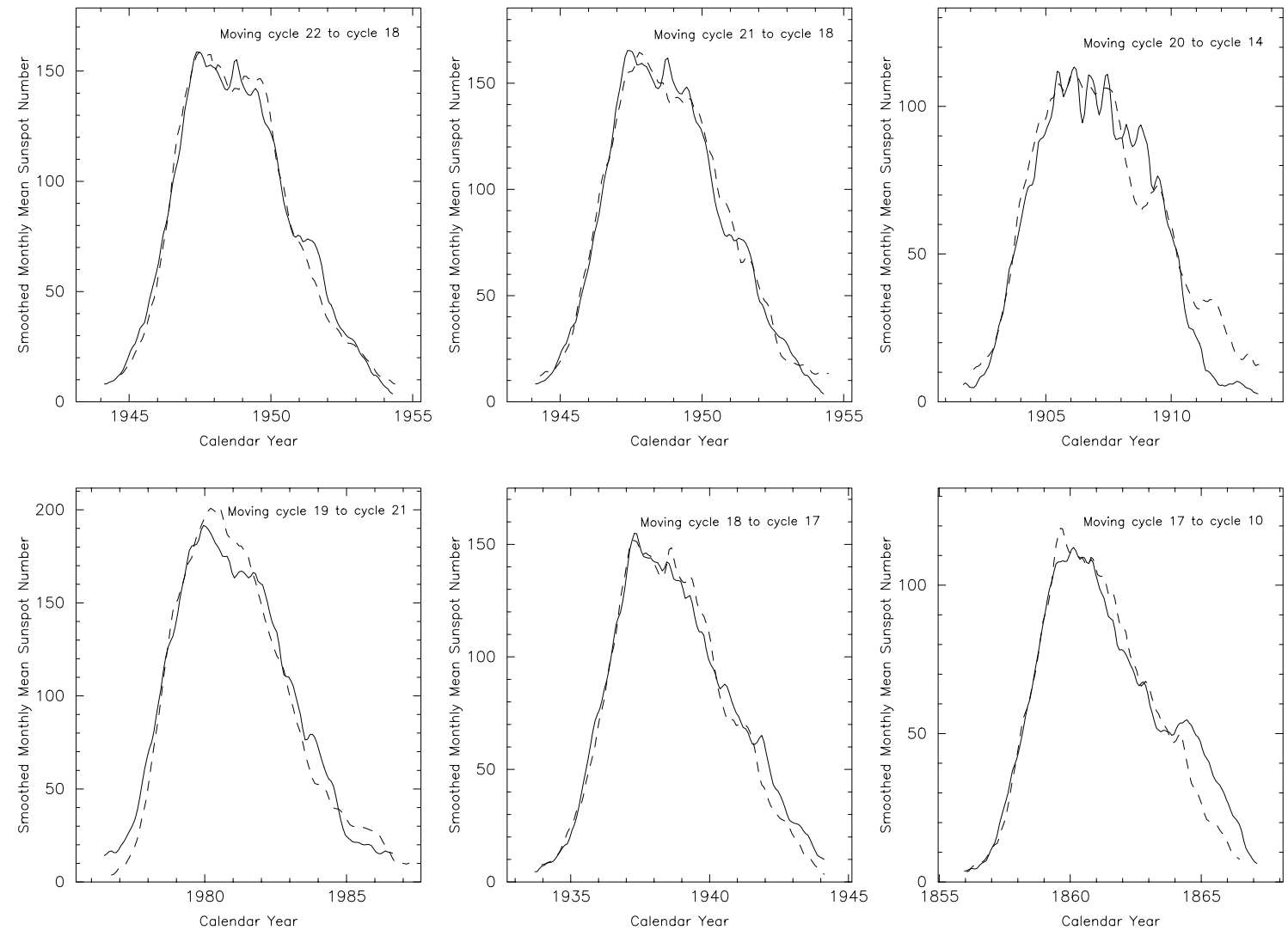

Fig. 6. Comparison of the moved cycles (the dashed lines) respectively with their similar cycles (the solid lines) which are multiplied by their determined parametric values $C$. 


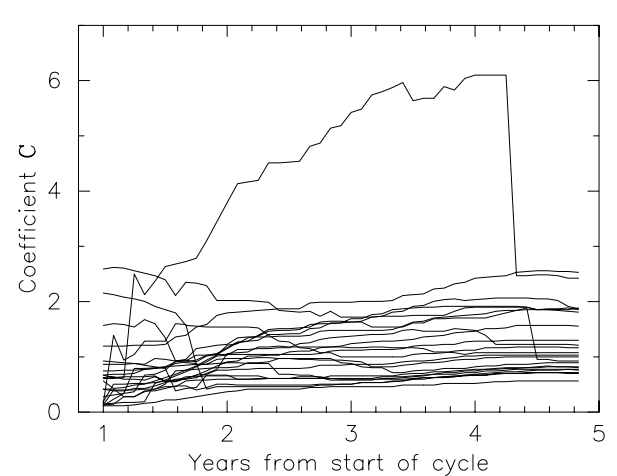

Fig. 7. Variation of the determined parameters $C$ with the used data points of cycle 23 when moving cycle 23 into the 22 complete cycles.

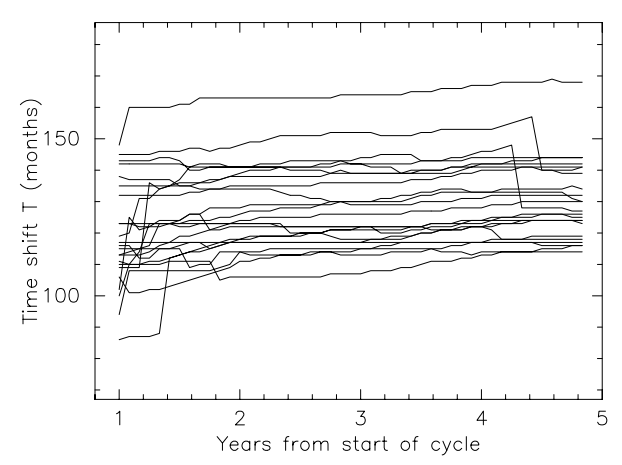

Fig. 8. Variation of the determined parameters $T$ with the used data points of cycle 23 when moving cycle 23 into the 22 complete cycles.

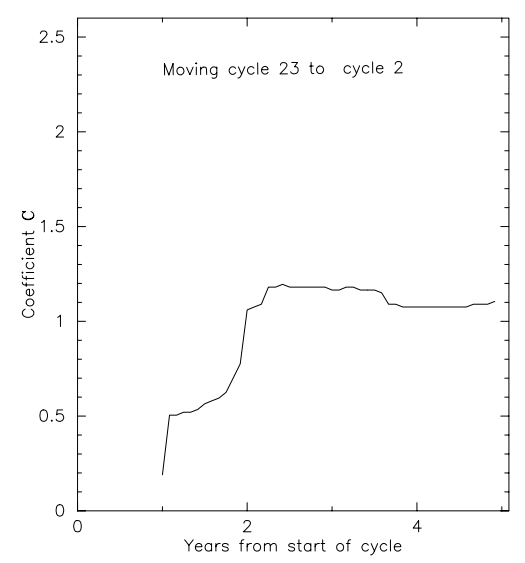

Fig. 9. Determinations of the parameter $C$ at one-month interval into cycle 23 when moving it into its similar cycle, cycle 2.

solar activity predicted by these authors. Because cycle 2 was of shorter period (Wilson et al. 1996), we infer here that cycle 23 also might be of shorter length (period less than 11 years), ending sometime in later 2006 or early 2007, well matching our result (Li et al. 2002).

\section{Conclusions and discussions}

Predicting the behavior of a sunspot cycle seems fairly reliable once the cycle is well underway, about 3 years after the minimum in sunspot number occurs (Hathaway et al. 1994). In this paper, this idea is tested, and the possibility of using sunspot

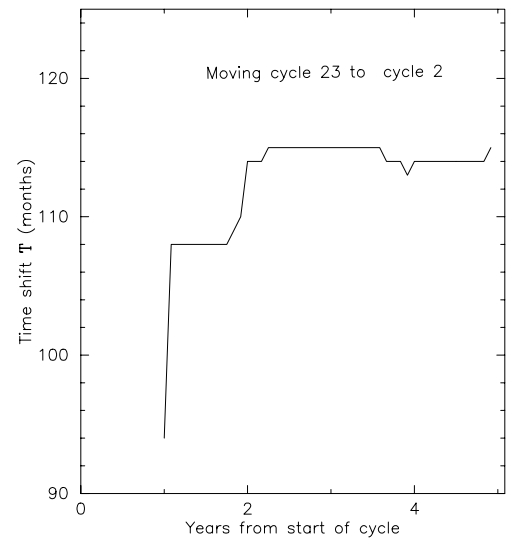

Fig. 10. Determinations of the parameter $T$ at one-month interval into cycle 23 when moving it into its similar cycle, cycle 2.

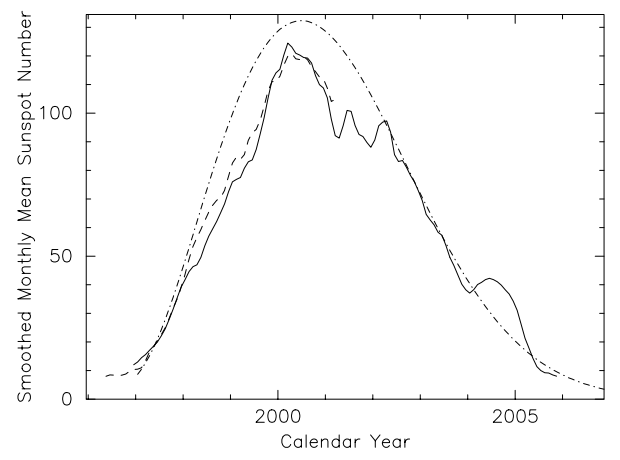

Fig. 11. Comparison of sunspot numbers of solar cycle 23 (the dashed line), those of its similar cycle (the solid line), and those predicted by MSFC Solar Physics Branch members (the dashed and dotted line).

numbers of the known solar cycles to predict the forthcoming solar activity of a progressing cycle is investigated. We find that the idea is practicable. The gross level of solar activity of a cycle can accurately estimated after 3 to 4 years following the beginning of the cycle, and with the use of sunspot numbers of a cycle within the first 3 to 4 years, and solar activity of the cycle in the ensuing several years can be accurately predicted. Solar activity of solar cycle 23 in the remaining years is predicted in this paper, and the gross level of solar activity of cycle 23 is estimated to be 1.08 times as much as cycle 2 or 0.75 times as much as cycle 22 .

Up to now, sunspot numbers have been available of 22 complete cycles. They are enough to allow us to utilise these 22 complete cycles, namely 22 "profiles" of solar activity to predict the forthcoming solar activity of a progressing cycle. On the other hand, as we know, the early record of sunspot sunspots (cycles 1 to 11 ) is of less accuracy and reliability than those that follow, it means that shapes of the early cycles are somewhat untrue. An analysis on comparisons against these early cycles certainly seems to invite criticism. We believe that, the more complete cycles of large reliability are, the larger is the possibility of using sunspot numbers of the known solar cycles to predict the forthcoming solar activity of a progressing cycle, and the more accurate the predicted solar activity of the progressing cycle should be. 
We have not known exactly the physics behind our proposed method. But two empirical principles are justified by the current investigation. First, for a given progressing solar cycle, there are always one previous cycle, whose behavior is mostly similar to the given cycle after its time profile been shifted and/or linearly multiplied by a constant. Secondly, as stated by Hathaway et al. (1994), the first 3 to 4 years behavior of a given solar cycle seems very indicative to the behavior of remainders of the cycle*. These two empirical principles may be used for the predictions of cyclic behavior of other solar and terrestrial activity indices ${ }^{1}$.

Acknowledgements. We would like to thank the referee for very useful and helpful comments. One of authors (Li K. J.) is grateful to the staff of SEES, Seoul National University for their hospitality. This work is supported by the National Science Foundation of China, the State Ministry of Science and Technology of China (NKBRSF G20000784), the Chinese Academy of Sciences, the Science Foundation of Yunnan Province, and the BK21 project of the Korean Government.

\section{References}

Harvey, K. L., \& White, O. R. 1999, JGR, 104(A9), 10759

Hathaway, D. H., et al. 1994, Sol. Phys., 151, 177

Hathaway, D. H., et al. 1999, JGR, 104, 22375

Hathaway, D. H., et al. 2001, Status of Cycle 23 Forecasts, in Space Weather, ed. P. Song, et al., Geophys. Monograph Ser., 125, 195 Kane, R. P. 2001, Sol. Phys., 202, 395

Lantos, P. 2000, Sol. Phys., 196, 221

Li, K. J., et al. 2001, A\&A, 368, 285

Li, K. J., et al. 2002, JGR, in press

Schrijver, C. J., \& Zwaan, C. 2000, Solar and Stellar Magnetic Activity (Cambridge University Press), 137

Schwabe, H. 1843, AN, 21, 233

Waldmeier, M. 1935, Astron. Mitt. Zurich, 14(133), 105

Waldmeier, M. 1939, Astron. Mitt. Zurich, 14(138), 470

Wilson, R. M. 1988, JGR, 93(A9), 10011

Wilson, R. M., et al. 1996, JGR, 101(A9), 19967

Wilson, R. M., et al. 1998, JGR, 103(A8), 17411

Wolf, R. 1852, Acad. Sci. Comp. Rend., 35, 704

\footnotetext{
1 Actually, Hathaway, Wilson, and Reichmann noted that the behavior of a sunspot cycle was well determined after the first two years of the cycle. It was then that one could fairly reliably determine the amplitude and shape of most sunspot cycles. Sunspot cycles have observed rise times of 3-5 years, from minimum to maximum, so once maximum has occurred, the remainder of the cycle appears easily determined, certainly to $10 \%$ or so.
} 